\title{
Transient 2D FEM-fluid network coupling for thermo-mechanical whole gas turbine engine simulations: modelling features and applications
}

\author{
Sabrina Giuntini ${ }^{1 *}$, Antonio Andreini ${ }^{1}$, Bruno Facchini ${ }^{1}$, Marco Mantero ${ }^{2}$, Marco $_{\text {Pirotta }}{ }^{2}$, \\ and Sven Olmes ${ }^{3}$ \\ ${ }^{1}$ Dept. of Industrial Engineering, University of Florence, Via di Santa Marta 3, 50139, Florence, Italy \\ ${ }^{2}$ Ansaldo Energia, Via Nicola Lorenzi 8, 16152, Genoa, Italy \\ ${ }^{3}$ Ansaldo Energia Switzerland, Römerstrasse 36, 5400, Baden, Switzerland
}

\begin{abstract}
In order to control the thermo-mechanical stresses that large heavy-duty power generation turbines have to face nowadays in their frequent operational transients, the analysis of the heat transfer between main flow, secondary air systems and structural components has to consider multi-physics coupled interactions, and has to be carried out with a whole engine modelling approach, simulating the entire machine in the real operating conditions. This is fundamental to guarantee a reliable assessment of life timing consumption and optimize clearances and temperature picks, through an efficient secondary air system design. It is here proposed a comprehensive description of modelling features and assumptions needed for the transient thermo-mechanical characterization of the whole engine through the application of a FEM-fluid network coupling methodology developed in collaboration with Ansaldo Energia and based on the open source code CalculiX ${ }^{\circledR}$. In the present work the transient thermal modelling capability of the procedure will be verified through its application to a real whole engine geometry under a realistic transient cycle, comparing results with those of a reference FEM code.
\end{abstract}

\begin{tabular}{|c|c|c|c|c|c|}
\hline \multicolumn{6}{|c|}{ NOMENCLATURE } \\
\hline$A$ & Area & {$\left[\mathrm{m}^{2}\right]$} & $L$ & Length & {$[\mathrm{m}]$} \\
\hline$c_{f r}$ & Friction factor & {$[-]$} & $M$ & Momentum & {$[\mathrm{J}]$} \\
\hline$c_{p}$ & $\begin{array}{l}\text { Specific heat at constant } \\
\text { pressure }\end{array}$ & {$\left[\mathrm{J} \mathrm{kg}^{-1} \mathrm{~K}^{-1}\right]$} & $\dot{m}$ & Mass flow rate & {$\left[\mathrm{kg} \mathrm{s}^{-1}\right]$} \\
\hline$C_{t}$ & Tangential gas velocity & {$\left[\mathrm{m} \mathrm{s}^{-1}\right]$} & $N, n$ & Generic amount & {$[-]$} \\
\hline d & Displacement vector & {$[\mathrm{m}]$} & $P$ & Pressure & {$[\mathrm{Pa}]$} \\
\hline$D_{h}$ & Hydraulic diameter & {$[\mathrm{m}]$} & $\dot{Q}$ & Thermal power & {$[\mathrm{W}]$} \\
\hline f & Body force vector per unit mass & {$\left[\mathrm{N} \mathrm{kg}^{-1}\right]$} & $q$ & Heat flux & {$\left[\mathrm{W} \mathrm{m}^{-2}\right]$} \\
\hline$F$ & Force & {$[\mathrm{N}]$} & $R$ & Radius & {$[\mathrm{m}]$} \\
\hline$h^{\phi}$ & Heat generation per unit mass & {$\left[\mathrm{W} \mathrm{kg}^{-1}\right]$} & $S R$ & Swirl ratio & {$[-]$} \\
\hline$H T C$ & Heat transfer coefficient & {$\left[\mathrm{W} \mathrm{m} \mathrm{m}^{-2} \mathrm{~K}^{-1}\right]$} & $t$ & Time & {$[\mathrm{s}]$} \\
\hline
\end{tabular}

\footnotetext{
* Corresponding author: sabrina.giuntini@htc.unifi.it
} 


\begin{tabular}{|c|c|c|c|c|c|c|c|c|}
\hline$T$ & Temperature & \multicolumn{2}{|l|}{$[\mathrm{K}]$} & Thick & \multicolumn{3}{|c|}{ Thickness } & {$[\mathrm{m}]$} \\
\hline $\mathbf{T}_{\mathrm{s}}$ & Thermal strain tensor & {$[-]$} & & $U$ & \multicolumn{3}{|c|}{ Rotational velocity } & {$\left[\mathrm{m} \mathrm{s}^{-1}\right]$} \\
\hline \multicolumn{9}{|c|}{ Acronyms } \\
\hline BCs & \multirow{2}{*}{\multicolumn{2}{|c|}{$\begin{array}{l}\text { Boundary Conditions } \\
\text { Finite Element Method }\end{array}$}} & \multicolumn{2}{|r|}{ SAS } & \multicolumn{4}{|c|}{ Secondary Air System } \\
\hline FEM & & & \multicolumn{2}{|r|}{ WEM } & \multicolumn{4}{|c|}{ Whole Engine Modelling } \\
\hline \multicolumn{9}{|c|}{ Greeks } \\
\hline$\epsilon$ & Elastic strain tensor & \multicolumn{3}{|l|}{$[-]$} & \multicolumn{3}{|c|}{ Stress tensor } & {$\left[\mathrm{N} \mathrm{m}^{-2}\right]$} \\
\hline к & Thermal conductivity tensor & \multicolumn{3}{|c|}{$\left[\mathrm{W} \mathrm{m}^{-1} \mathrm{~K}^{-1}\right]$} & \multirow{2}{*}{\multicolumn{3}{|c|}{ Rotational Speed }} & {$\left[\mathrm{rad} \mathrm{s}^{-1}\right]$} \\
\hline$\rho$ & Density & \multicolumn{3}{|c|}{$\left[\mathrm{kg} \mathrm{m}^{-3}\right]$} & & & & \\
\hline \multicolumn{9}{|c|}{ Subscripts } \\
\hline$a b s$ & Absolute & face & \multicolumn{3}{|c|}{ Face } & plane & \multicolumn{2}{|c|}{ Plane } \\
\hline ass & Assigned & $f r$ & \multicolumn{3}{|c|}{ Friction } & real & \multicolumn{2}{|c|}{ Real } \\
\hline axi & Axisymmetric & hole & \multicolumn{3}{|c|}{ Hole } & rel & \multicolumn{2}{|c|}{ Relative } \\
\hline blade & Blade & $i j k$ & \multirow{2}{*}{\multicolumn{3}{|c|}{-th generic entity }} & $t m$ & \multicolumn{2}{|c|}{ Thermal mass } \\
\hline$c$ & Compressor & in & & & & $w$ & \multicolumn{2}{|c|}{ Wall } \\
\hline $\operatorname{conv}$ & Convective & $\max$ & \multicolumn{3}{|c|}{ Maximum } & wind & \multicolumn{2}{|c|}{ Windage } \\
\hline$c w r$ & Correction for wall rotation & $\min$ & \multicolumn{3}{|c|}{ Minimum } & 0 & \multicolumn{2}{|c|}{ Total quantity } \\
\hline elem & Element & out & \multicolumn{3}{|c|}{ Outlet } & 1,2 & \multicolumn{2}{|c|}{ Generic inlet/outlet } \\
\hline$f$ & Fluid & $p$ & Pun & nping & & & & \\
\hline
\end{tabular}

\section{Introduction}

Controlling gaps and clearances affects engine efficiency and component failures. Achieving effective secondary air system (SAS) design means to fulfil all the functionalities required to the secondary air system (cooling, sealing, purging, etc) with the minimum air consumption, in order to limit the penalty on the cycle performances. Air consumption is directly affected by mass flow splits and pressure losses, and ultimately, by sealing gaps and cross section areas of flow passages. The weight of these topics, always particularly relevant for aeronautical engines, increases today also in the heavy-duty gas turbines field. Indeed, engines for electric power generation are nowadays subjected to very frequent start-ups, shutdowns and load adjustments, primarily due to the energy market privatization and today even more for the renewable energy development. Gas turbine plants which can be dispatched within minutes are important assets for balancing electric system loads and maintaining grid reliability. Transient operation should be very fast, in order to achieve rapidly the required load, circumstance that causes strong changes in temperature that components must face, which are responsible for significant deformations of geometries and thermo-mechanical stresses. Managing such complex systems and multi-physic phenomena can be possible only developing a procedure that involves all the components of the engine and allows its comprehensive simulation. According to this, a Whole Engine Modelling (WEM) approach for the prediction of the engine operation in transient conditions is necessary to monitor the overall thermo-mechanical behaviour of the engine and achieve a functional design able to control the expansion rates of parts and ensure good seals, guaranteeing integrity and efficiency of the whole system.

Due to the complexity of phenomena and the consequent high computational cost of $2 \mathrm{D}$ and 3D CFD simulations, in the conventional industrial practice is common to use different codes tailored to the solid and fluid domains separately, allowing to exploit the advantages of applying specific numerical approaches and models. The conjugate heat transfer problem is generally solved with dimensionally heterogeneous approaches [1], using FEM analyses coupled with 1D flow network solvers to evaluate fluid-metal temperatures and thermomechanical stresses, with a considerable reduction of the computational cost. In literature it is possible to find works dealing with commercial 1D flow and FEM solvers [2, 3], or 
exploiting proprietary tools $[1,4]$ or even based on open source codes $[5,6]$. Works $[7,8]$ of the authors of the current paper are based on an open source code too, and introduced a first insight in the partly coupled FEM-1D fluid network thermo-mechanical procedure here presented, a project carried out in collaboration with Ansaldo Energia. The methodology has then developed and enriched of the inclusion of the effect of the running clearances on the secondary air system properties, applying the overall procedure to a real engine model in [9]. In the present paper authors wants to focus and deep the modelling features and applications of the transient 2D FEM-fluid network coupling procedure for thermo-mechanical analyses included in the overall WEM aero-thermo-mechanical approach presented in [9].

\section{Aero-thermo-mechanical methodology description}

The WEM aero-thermo-mechanical methodology developed by the authors in collaboration with Ansaldo Energia [9], aims at catching the transient aero-thermo-mechanical behaviour of the engine, appreciating the coupled phenomena occurring among the different multiphysicals fields, i.e. the effects of gaps and clearances redefinition on the SAS properties because of the heat transfer. This represents a novelty for large power generation engines, for which traditionally the effect of the interaction between running clearances and fluid properties is not considered in transients, and the sequential solution of SAS and thermomechanical analyses is carried out in one single finalizing iteration. In the present approach, differently from the conventional practice, the SAS solution is not solved once and scaled for all the other time steps, but there is an update of SAS properties according to the effect of heat transfer and solid deformations.

The procedure is based on the solution of an iterative process between the thermostructural analysis of the solid-fluid domain and the aerodynamic solution of the SAS fluid network. Fluid-metal aero-thermal interactions are handled therefore by a partly coupled scheme, since SAS aerodynamic and FEM analyses are performed separately. The thermomechanical solution is carried out with a customized version of the open source FEM solver CalculiX $^{\circledR}$. The SAS solution is obtained with a customized version of the native open source CalculiX ${ }^{\circledR}$ one-dimensional fluid network solver. Each sub-model is run standalone carrying out a simulation covering the entire operation and only after the evaluation of the last time step of the whole cycle, its solution is imposed as boundary condition for the other sub-model. The transient heat transfer problem over the solid domain is coupled to a sequence of structural static and steady flow problems using a quasi-steady state approximation. The very high difference in the convection and conduction time scales allows to consider a time scale separation between the two phenomena [9], assuming that the "fast" fluid transients, during which the "slow" metal temperature state is assumed to stay constant, can be neglected [10].

Following the flow chart of the iterative scheme (Fig. 1), first there is an initialization of the variables, which in general corresponds to the cold engine conditions, even though whatever initial temperature and geometrical fields can be imposed as initial operation status (cold, warm or hot) and used to start the calculation process. Characteristic SAS geometrical features (hydraulic diameters, lengths and gaps) are therefore set at desired initial values (violet block). These values, corresponding in the FEM model to the initial distance between 2 nodes on either side of the characteristic length, are assumed as reference. During the simulation their displacements are evaluated to assess the variation of their position respect to the initial conditions. After setting initial values, a first adiabatic solution of the standalone aerodynamic model of the SAS (red block) is carried out through a sequence of steady state solutions covering the entire cycle, imposing main flow annulus and inlet SAS conditions as $\mathrm{BCs}$ once and for all at the beginning of the calculation. Then, the first obtained SAS solution, characterizing as time series the fluid properties (mass flow rates, pressures and initial inlet temperatures), is imposed as BCs in the thermo-mechanical model (blue block) by means of 


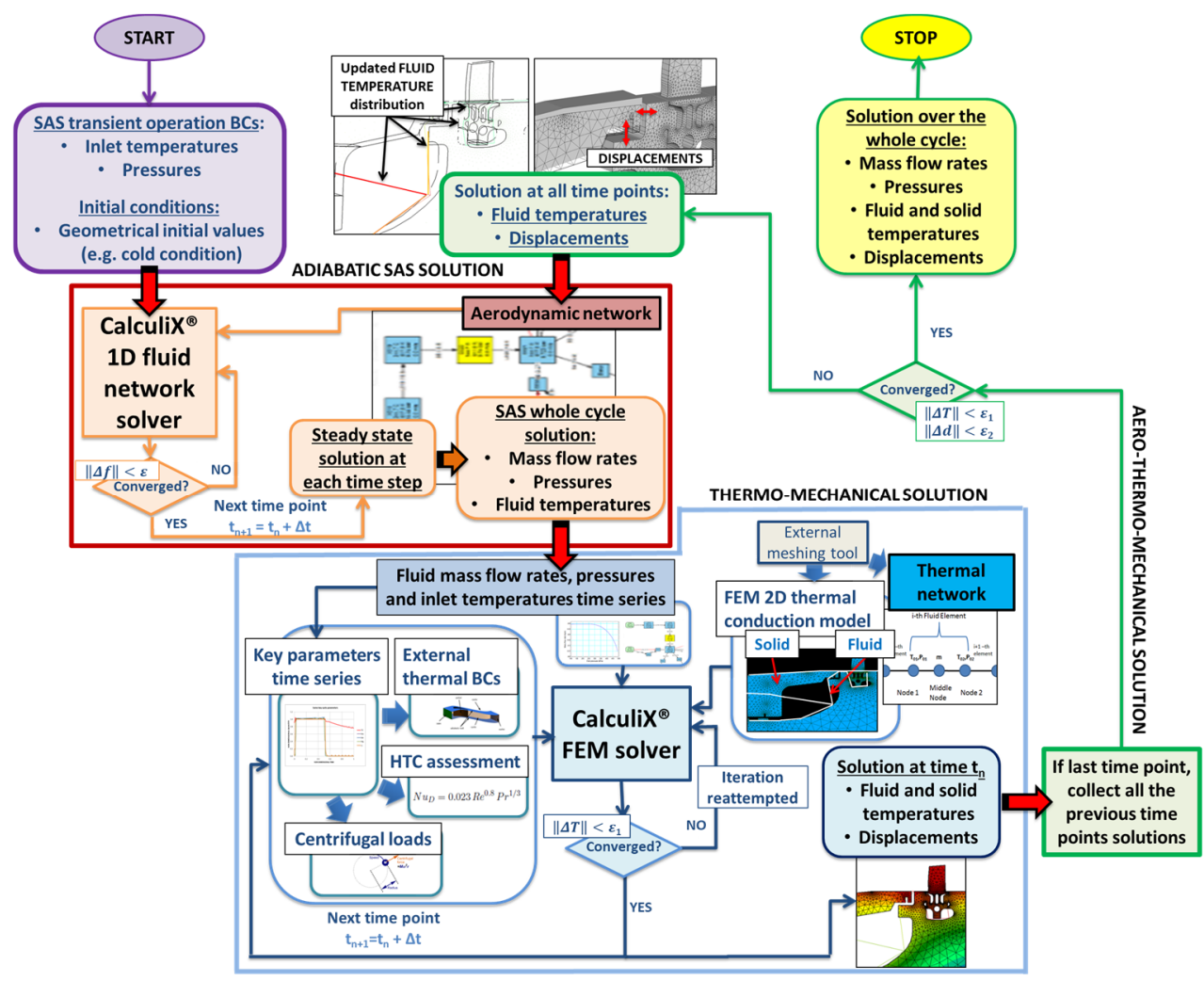

Fig. 1. Aero-thermo-mechanical methodology scheme.

a dedicated thermal network for the evaluation of the heat transfer between fluid and solid. Applying all the other BCs necessary to complete the thermo-mechanical model, at each time step fluid-metal temperatures and displacements are stored step by step as the thermomechanical solution proceeds. Due to the heat transfer, the predefined monitor nodes have modified their relative position, subjected to axial and radial displacements, along with the rest of the mesh nodes. At the end of the transient cycle, once the last time step has been performed, temperatures and displacements are post-processed and compared with the values obtained at the previous overall iteration on the transient operation (green block). If convergence is not reached, temperatures and displacements are post-processed and used to update the geometries of the secondary air system and to impose new levels of temperature in the subsequent SAS standalone solution, as new BCs. After performing this new SAS analysis, the recalculated mass flow and pressure distributions are therefore applied in the thermo-structural simulation for a new iteration, with a loop between thermo-mechanical model and aerodynamic fluid network, repeated until a converged solution is reached (yellow block). In this way, the effects of the solid-fluid heat transfer and running clearances on the mass flow splits, pressure drops and temperatures in the fluid network, are accounted. More details about the SAS solution and the overall WEM approach can be found in [9].

\section{Transient 2D FEM-fluid network coupling approach for thermo- mechanical WEM analyses}

The aim of the present work is focus on the thermo-mechanical procedure of the aero-thermomechanical methodology described in the previous Section, whose early development can be 
found in $[7,8]$. The paper wants to provide a more comprehensive discussion about the main fundamental modelling features introduced to manage thermo-mechanical WEM simulations, and show the consistency of the results of its application to a real engine model.

\subsection{Thermo-mechanical solution overview}

The thermo-mechanical calculation (see blue block in Fig. 1), performed with the FEM solver CalculiX $^{\circledR}$, applies a unique code and needs a single model for the thermal and mechanical solutions. The procedure, developed for the solution of the behaviour of a whole engine cross section, foresees that the solid domain is modelled with a 2D finite element axisymmetric approach with proper modelling for the treatment of 3D features. The FEM model includes a dedicated 1D thermal fluid network through which fluid-metal heat transfer is computed.

As reported in [11], the energy equation is solved considering temperature and flux boundary conditions. The governing equation can be written as:

$$
\boldsymbol{\nabla} \cdot(-\boldsymbol{\kappa} \cdot \boldsymbol{\nabla T})+\rho c_{p} \frac{\partial T}{\partial t}=\rho h^{\phi}
$$

where $T$ is the temperature, $\boldsymbol{\kappa}$ contains the conduction coefficients, $\rho$ is the density, $h^{\phi}$ the heat generation per unit of mass and $c_{p}$ is the specific heat $[11,12]$.

Temperatures and fluxes imposed as boundary conditions, consider also the application of radiative and convective BCs. The latter is expressed as:

$$
q=H T C \cdot\left(T_{w}-T_{f}\right)
$$

where $q$ is the magnitude of the heat flux normal to the surface, HTC is the heat transfer coefficient, $T_{w}$ is the wall temperature and $T_{f}$ is the fluid temperature. Referred to a single fluid element, Eq. 2 can be rewritten as in Eq. 3, and together with Eq. 4, applying mass flow rates and boundary temperatures of the secondary air system, they describe the fluid-solid coupling in the thermo-mechanical solution due to the heat transfer, where the downstream extremity node of the 1D fluid element (the $\mathrm{j}$-th node, supposing the $\mathrm{i}$-th as the upstream one) in the FEM model will be affected by the heat load $\dot{Q}_{i, j}$, according to the following equations:

$$
\begin{gathered}
\dot{Q}=H T C \cdot A \cdot\left(T_{w}-T_{f, i}\right) \\
\dot{Q}_{i j}=\dot{Q}=c_{p} \cdot \dot{m}_{i j} \cdot\left(T_{f, j}-T_{f, i}\right)
\end{gathered}
$$

Structural coupling foresees that pressure from SAS calculation is used in the FEM model for the application of mechanical loads regarding the action of the static fluid pressure on the solid. Concerning the elastic problem in the solid domain, the governing law is of the form:

$$
\rho \frac{\partial^{2} \mathbf{d}}{\partial t^{2}}-\nabla \cdot \sigma\left(\epsilon, \mathbf{T}_{\mathbf{s}}\right)=\rho \mathbf{f}
$$

where $\mathbf{d}$ is the displacement vector, $\boldsymbol{\sigma}$ is the stress tensor depending on the linear elastic strain tensor $\boldsymbol{\epsilon}$ accounting for the mechanical strain, and on the thermal strain tensor $\mathbf{T}_{\mathbf{s}}$ caused by the variation of the temperature $\Delta T$ from its original value and modelling thermal expansion [1], while $\mathbf{f}$ is the vector of body forces per unit of mass acting on the solid.

In the proposed procedure, during the thermo-mechanical calculation, there is a sequential solution (totally hidden to the user) of the thermal and structural problems above. For each time increment a thermal analysis is performed first. Then, the resulting temperature field is used as boundary condition for a subsequent mechanical analysis for the same time increment. This choice, neglecting effects of displacement on the view factors, leads to a system of equations which is smaller and faster, and execution time can be saved.

Overall, in order to perform the calculation, the inputs provided to the thermo-mechanical solver are (see blue block in Fig. 1): 


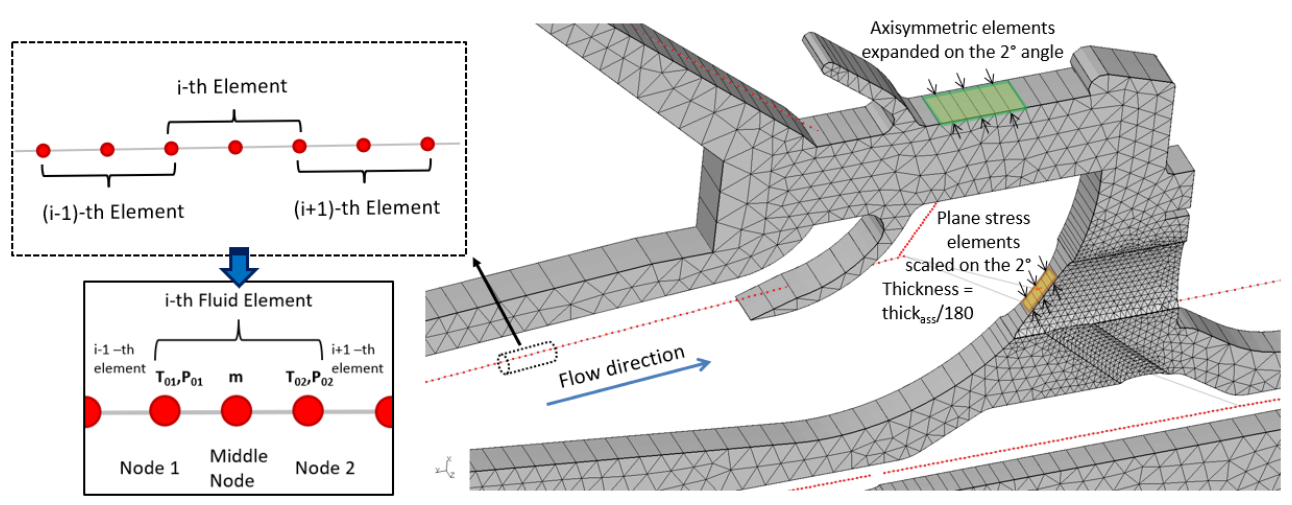

Fig. 2. Thermal network in the FEM model.

- $\quad$ solid and fluid mesh (unique model);

- $\quad$ SAS properties (mass flow rates, pressure distributions and inlet temperatures);

- other prescribed quantities varying in time (key parameters);

- centrifugal loads;

- displacements constraints;

- thermal BCs.

The application of the above listed inputs is treated in the following Sections, clarifying how a typical WEM thermo-mechanical simulation is set in the present methodology.

\subsection{Fluid network features}

In CalculiX ${ }^{\circledR}$ the fluid network is directly integrated in the thermo-mechanical FEM model. Triplets of nodes are inserted in the domain, where each triplet represents a fluid element. Pressure and temperature values are stored in the extremity nodes, while mass flow information in the middle side node (see details in Fig. 2). The concatenation of triplets constitutes the thermal network.

The thermal network used in the FEM model is dedicated for the FEM application and it is basically different from the aerodynamic one that could be used for the SAS solution. First, the aerodynamic network foresees that mass flow, pressure and temperature are unknown of the generic fluid element and must be solved. On the contrary, the thermal network involves the temperature solution only, since mass flow and pressure distributions are imposed as BCs. Secondly, there are also differences in the spatial discretization. The aerodynamic case discretization is in general coarser than the thermal one, mainly set by the subsequent change in typology and geometry of the elements along the SAS structure, independently from the discretization of the solid domain. On the contrary, in the thermal case, the number of elements to be inserted in the fluid network is decided by the spatial configuration of the solid mesh and by the complexity of the thermal phenomena involved. With the view to include the thermo-mechanical procedure in a WEM aero-thermo-mechanical methodology (as in Section 2), it could be thought to solve thermal and aerodynamic problems simultaneously within the same model, simply adjusting the coarse fluid mesh (aerodynamic) to the finest one (thermal), and adopting this latter kind of discretization for the common model. However, due to the notorious difficulty to converge of the highly nonlinear equations of the fluid aerodynamic network, increasing the number of fluid elements in attempt to provide spatial convergence would cancel out any computational advantage of the monolithic approach. This is way the choice of maintaining separated the aerodynamic and thermal network appears as the most suitable (more details about fluid networks types can be found in [9]). 


\subsection{Boundary conditions}

Mass flow rates and pressure distributions from a previous SAS solution are applied to the thermo-mechanical model as boundary conditions, and potentially they can be previously obtained from any fluid network solver providing mass flow splits and pressures. In most standard industrial practices, these flow boundary conditions, determined at specific time points of the operation (generally at the steady state condition), are scaled based on the standard cycle key parameters and aerodynamic data (e.g. rotational speed and power), and expressed as a function of time in order to reproduce the overall transient cycle conditions. In the methodology presented in Section 2, SAS properties come from adiabatic standalone simulations performed through the CalculiX ${ }^{\circledR}$ 1D network solver, by means of the solution of an aerodynamic model of the network. The SAS solution is not solved once and scaled for all the other time steps, but through the iterative loop, standalone SAS calculations are performed until convergence, considering the heat transfer and the deformations.

Convective coefficients and heat pickup (such as that due to windage power generation) can be expressed as function of significant parameters (assigned in their turn as BCs) or of runtime calculated quantities. These expressions are mainly based on empirical correlations customized for gas turbine applications and are evaluated runtime by the solver.

Main flow BCs are generally based on preliminary analysis and on engine test data when available. They are imposed based on key parameters and eventually scaled in time.

Radiation heat transfer is imposed in all those regions where high thermal gradients are supposed, thermal contacts are set between all the surfaces with contact. Centrifugal loads are imposed on rotoric components, structural constraints are applied on the extremities of statoric parts, and both are exposed to the pressure loads expressed as explicit distributed loads BCs or deriving from the pressure exercised by the fluid network on the solid.

\subsubsection{Thermal BCs types}

Main flow, SAS and experimental/field data can be assigned in different ways.

So-called convecting zones simulate the convection from a solid boundary to a fluid of a known temperature. Fluid temperature and heat transfer coefficient must be specified, addition rotational effects can be defined. No fluid elements are created in the FEM model.

Thermal streams model the case the element is adjacent to a moving fluid whose temperature is also unknown. Fluid elements are created. Mass flow rates, inlet air temperatures, heat transfer coefficients, heat pickup and rotational effects, if present, must be specified. The inlet air temperature can be dependent on specified performance data of the engine (key parameters) and/or can be referred to inlet/outlet temperatures from other thermal $\mathrm{BCs}$ to which the stream is virtually linked, through complex formulations too.

Thermal contacts simulate heat flow across a contact pair due to the temperature difference between the corresponding slave and master surfaces. Conductance can be defined as a function of key parameters such as the rotation speed or of runtime evaluated values such as the contact pressure and the mean surfaces temperature.

Finally, imposed temperatures distributions can be assigned on the mesh nodes.

\subsection{Axisymmetric approach}

The procedure is devised for the solution of a fully featured 2D axisymmetric gas turbine whole engine model. Axisymmetric approach has the great advantage of containing computational costs, making possible calculations otherwise infeasible with a fully $3 \mathrm{D}$ approach. Stator and rotor components are modelled as axisymmetric, exception for the zones of the mesh corresponding to channels, holes, bores, blades and nozzles. Since the geometry 


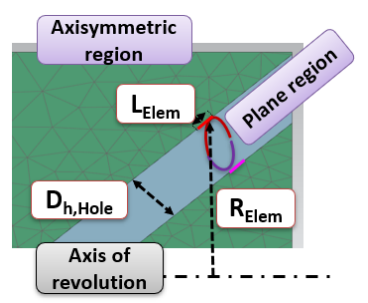

(a) Bore in the 2D domain.

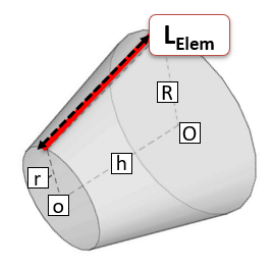

(b) Axi element edge revolution on the $360^{\circ}$.

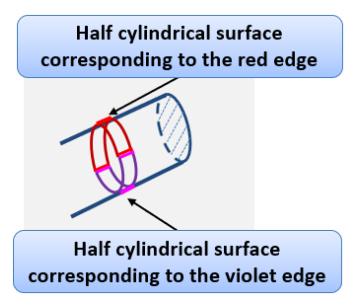

(C) Real area associated to each side element of the portion.

Fig. 3. Modelling 3D features in the 2D domain - bores.

is modelled in 2D, suitable area and volume multipliers are considered for all nonaxisymmetric features. These letters are treated with plane stress elements with an equivalent wall thickness and appropriate modelling assumptions to account for $3 \mathrm{D}$ features.

CalculiX $\mathrm{X}^{\circledR}$ uses on the 2D model solution an expansion angle fixed at $2^{\circ}$ (see thickness in Fig. 2). Mass flow and plane element thickness are scaled automatically by the solver in order to consider the proper fraction of materials on the expansion angle. This affects the HTC and heat pickup assessments, which in they turn must be scaled. Scaling is also necessary because of the difference between real surfaces and surfaces available in the FEM model for the loads application in the interfaces between plane and axisymmetric elements, which must be carefully considered in order to respect the energy balance in the 3D phenomena approximation with $2 \mathrm{D}$ modelling. Automatic scaling of quantities was missing in the Calculi $\mathrm{X}^{\circledR}$ original code and it has been added in the presented procedure through dedicated implementations. Different cases of interfaces and consequent scaling must be faced in a WEM procedure. Below, the main typologies are reported with simplified examples for the sake of brevity. It will be referred to the HTC scaling, but the same considerations apply for heat pickup, and all quantities applied as distributed loads on surfaces.

\subsubsection{Scaling on plane elements - Blades}

On a blade row the globally assigned thickness on the corresponding plane elements is the thickness of the single blade by the number of blades in the row. Considering the thickness and mass flow scaling, and considering that the surface of application of the HTC coefficient on a blade correspond to the sum of the $2 \mathrm{D}$ plane faces, the $\mathrm{HTC}$ to be assigned is:

$$
H T C_{\text {face }}=\left(\left(H T C_{\text {blade }} \cdot N_{\text {blade }}\right) / 180\right) / 2
$$

where $H T C_{\text {blade }}$ is the $\mathrm{HTC}$ value on the $2 \mathrm{D}$ blade surface which must be scaled on the plane element expansion on the $2^{\circ}$ dividing by 180 , and additionally scalded dividing by 2 in order to obtain the proper value to apply on the single rear and front face of the plane element.

\subsubsection{Scaling in axisymmetric-plane elements coupling - Holes}

In case of bores and holes the elements thickness applied is the result of the subtraction from the solid of the empty volume of the single hole multiplied for the global number of holes. This generates an interface between plane and axisymmetric regions on which the thermal load can be applied (Fig. 3a). In the procedure the choice of apply the heat load on the axisymmetric element surface has been made. In CalculiX ${ }^{\circledR}$ this means that the surface on which the HTC value will be applied is the surface obtained from the revolution of the edge on the $360^{\circ}$ (lateral surface of a truncated cone, Fig. 3b). Obviously, this surface does not coincide with the real one, which corresponds instead to the inner surface of the single bore 


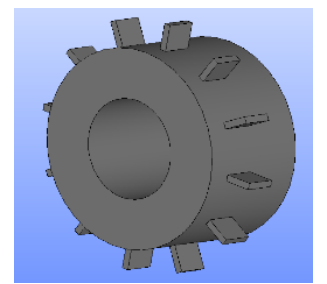

(a) Rotor with blades in 3D.

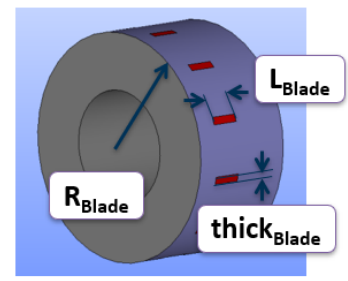

(b) Blade thickness on the endwall.

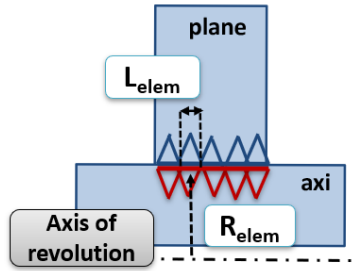

(C) 2D axi-plane interface.

Fig. 4. Modelling 3D features in the 2D domain - endwalls.

multiplied for the number of the bores. Hence, there is the need to properly scale the HTC coefficient in order to maintain the correct product $A \cdot H T C$. Referring to the area of a single element of the mesh, this follows:

$$
H T C_{\text {axi }} \cdot A_{\text {axi,elem }}=H T C_{\text {real }} \cdot A_{\text {real }}
$$

where $H T C_{a x i}$ is the scaled value to be applied in the CalculiX ${ }^{\circledR}$ model in order to consider the surface of application $A_{\text {axi,elem }}$ used by the solver, while $H T C_{\text {real }}$ is the real value which should be applied on the inner surface of the ducts. Considering $A_{\text {real }}$ for each element of length $L_{\text {Elem }}$ in the bore as the half lateral surface of the cylinder of height $L_{\text {Elem }}$ (Fig. 3c), Eq. 7 becomes:

$$
H T C_{\text {axi }} \cdot 2 \pi \cdot R_{\text {elem }} \cdot L_{\text {elem }}=H T C_{\text {real }} \cdot\left(2 \pi \cdot R_{\text {hole }} \cdot L_{\text {elem }}\right) / 2
$$

where $R_{\text {Elem }}$ is the average radial quota of the element, $L_{\text {Elem }}$ is the length of the edge of the $2 \mathrm{D}$ element at stake (located at the interface between plane and axisymmetric domains), and $R_{\text {hole }}$ is the radius corresponding to the hydraulic diameter of the hole $D_{h, \text { hole }}$. Considering the total number of holes $N_{\text {hole }}$ in the region, the value to be applied is:

$$
H T C_{\text {axi }}=H T C_{\text {real }} \cdot \frac{R_{\text {hole }} / 2}{R_{\text {elem }}} \cdot N_{\text {hole }}
$$

\subsubsection{Scaling in axisymmetric-plane elements coupling - Endwalls}

Consider a rotor equipped with blades (Fig. 4a). If user wants to impose an HTC value in the region between a blade and the other (Fig. 4b), it is necessary to take in mind that applying the HTC on the edge delimiting the blade from the rotor in the 2D model on the axisymmetric side (Fig. 4c), the solver will consider as area of exchange the global surface of revolution on the $360^{\circ}$, while the surface over which the endwall HTC value should act is the $2 \pi R_{\text {blade }} L_{\text {blade }}$ reduced of the surface of the single blade hub, multiplied for the number of blades. Therefore, applying again Eq. 7, the scaling formulation of the HTC coefficient on endwalls at the interface between axisymmetric rotor elements and blades plane elements is:

$$
H T C_{a x i}=H T C_{\text {real }} \cdot \frac{\left(2 \pi \cdot R_{\text {elem }}-\text { Thick }_{\text {elem }} \cdot N_{\text {blade }}\right)}{2 \pi \cdot R_{\text {elem }}}
$$

\subsubsection{Scaling at plane-plane interfaces}

Consider now an interface with plane elements with different thicknesses (e.g. between the blade and its platform), and fluid elements exchanging heat with the free surface at the interface. Choosing to apply the heat load on the thicker element, however it has to be considered that the real surfaces of exchange for the $\mathrm{BC}$ is the difference between the different thicknesses of components, so that the proper scaling required is of the form: 

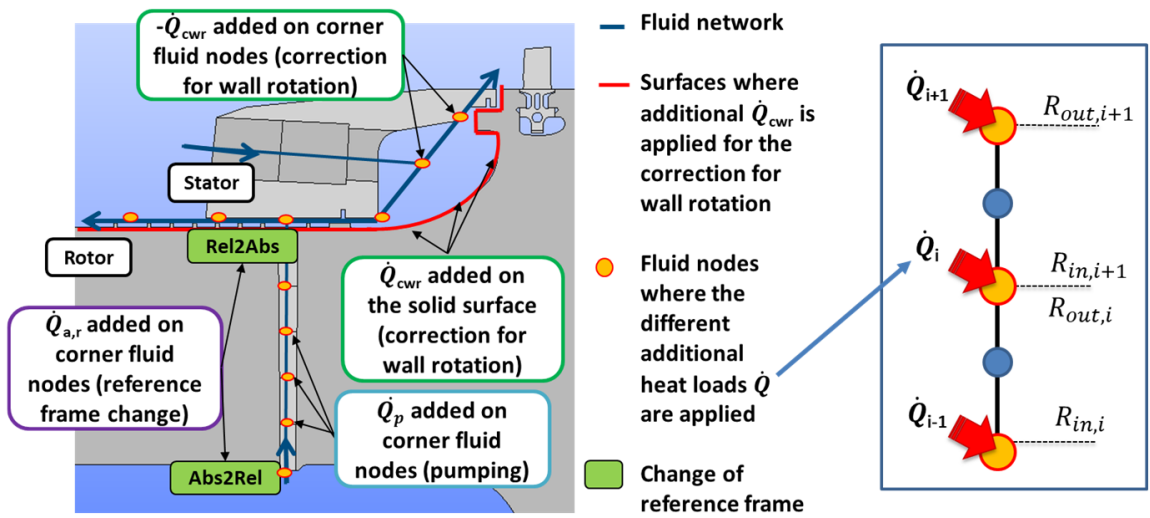

Fig. 5. Rotational effects handling - simplified overview.

$$
H T C_{\text {plane }}=H T C_{\text {real }} \cdot \frac{\left(\text { Thick }_{\text {max }, \text { elem }}-\text { Thick }_{\text {min,elem }}\right)}{\text { Thick }_{\text {max,elem }}}
$$

where $H T C_{\text {plane }}$ is the HTC value applied on the thicker element, while Thick $k_{\text {max elem }}$ and

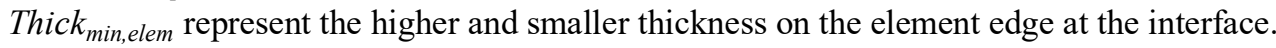

\subsection{Rotational effects handling}

Effects of rotation are considered applying equivalent additional heat loads on fluid nodes and eventually on solid elements, according to the type of phenomena (Fig. 5). Formulations derive from the conservation of rotalpy, relations in velocity triangles and definition of total absolute/relative quantities (details can be found in [7]). A generalized treatment of rotational effects was missing in the CalculiX ${ }^{\circledR}$ original code, so customizations have been introduced.

Referring to the Table 1, the relative reference frame and the consequent effects of pumping, are considered adding to the fluid nodes the heat load characterized by Eq. 12 and 13. In case of change of reference system, the Eq. 14 thorough Eq. 15 is applied on the fluid node at the radius at which change happens. In case of fluid network in an absolute reference frame exchanging heat with both static and rotating surfaces (as in the case of stator-rotor interfaces), a correction for wall rotation must be applied on the rotor side and on the fluid nodes involved. The heat exchanged with the rotating surface can be expressed as the sum of two contributions (Eq.16). Eq. 17 expresses the contribution solved automatically in the absolute reference frame, while Eq. 18 is the correction for the wall rotation which is imposed through a distributed heat load on each k-th mesh element involved and it is applied with opposite sign to the $\mathrm{j}$-th fluid node located at the $R_{k}$ radius.

Table 1. Rotational effects-related equations.

\begin{tabular}{|cc|c|}
\hline Characteristic heat load & Main driving quantity \\
\hline$\dot{Q}=\dot{m} \cdot c_{p} \cdot \Delta T_{0, r e l}(R)$ & $(12)$ & $\Delta T_{0, r e l, i}=\omega^{2} \cdot \frac{\left(R_{o u t, i}^{2}-R_{i n, i}^{2}\right)}{2 c_{p}}$ \\
\hline$\dot{Q}=\dot{m} \cdot c_{p} \cdot \Delta T_{0, a b s / r e l}(R)$ & $(14)$ & $T_{0, a b s}-T_{0, r e l}=-\frac{U^{2}-2 U C_{t}}{2 c_{p}}$ \\
\hline $\begin{array}{c}\dot{Q}=H T C \cdot A \cdot\left(T_{w}-T_{0, r e l, f}\right) \\
=Q_{\text {conv }, 1}+Q_{\text {conv }, 2}\end{array}$ & $(16)$ & $Q_{\text {conv }, 1}^{\cdot}=H T C \cdot A \cdot\left(T_{w}-T_{0, a b s, f}\right)$ \\
\cline { 2 - 3 } & & $Q_{c o n v, 2, k}=H T C_{k} \cdot A_{k} \cdot\left(\frac{U_{k}^{2}-2 U_{k} C_{t, j}}{2 c_{p}}\right)$
\end{tabular}



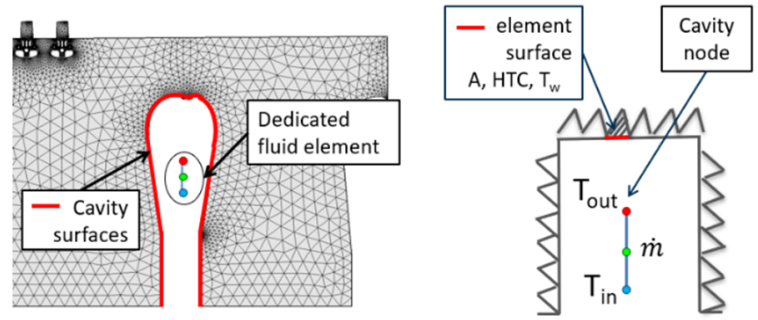

Fig. 6. Thermal mass handling scheme.

\subsection{Heat pickup}

Heat pickup effects are modelled with the application of an additional heat load on the fluid nodes, similarly to what done for modelling rotational effects. This functionality was not present in the original version of Calculi $\mathrm{X}^{\circledR}$ and it has been included with a dedicated implementation. As in the case of HTC, heat pickup can be evaluated through formulations or empirical correlations that user can insert in the simulation. It is used for some modelling aspects, among them, effects of windage due to surfaces rotation, producing a temperature increase of the air that flows through rotor-stator interfaces such as labyrinth seals.

Referring to the formulation of the friction force:

$$
F_{f r}(R)=\frac{c_{f r} \rho}{2}(1-S R(R))^{2} \cdot R^{2} \cdot \omega^{2} \cdot A
$$

through the momentum $M_{f r}(R)$, windage can be expressed as:

$$
Q_{\text {wind }}(R)=M_{f r}(R) \cdot \omega=F_{f r}(R) \cdot R \cdot \omega=\frac{c_{f r} \rho}{2}(1-S R(R))^{2} \cdot R^{3} \cdot \omega^{3} \cdot A
$$

where $R, A$ and $c_{f}$ are respectively the radius location, the area of the solid element and the friction factor over the area, $\rho$ is the density of the fluid element involved, while $S R=C_{t} / U$ and $\omega$ are the swirl ratio at the corresponding radius and the rotation velocity, respectively. Hence, the windage term $Q_{\text {wind }}$, responsible for the fluid temperature augmentation due to the action of the solid walls on the fluid, is added to the fluid node as an additional heat load.

\subsection{Thermal masses}

The engine structure is characterized by the presence of manifold cavities. Some of them are not fed by actual airflows and an effective fluid motion on walls cannot be defined. This case of flow branch is not modellable with a conventional thermal stream since no effective mass flow efflux can be identified neither the fluid temperature is known. To this end, a dedicated implementation has been introduced in the fluid network generation, foreseeing a disjoined fluid element whose BCs are differently set according to the phenomena to be modelled.

In case fluid motion cannot be defined and the enclose thermal mass exchanges heat with the metal surfaces contributing to mediate the temperature over the same walls, considering the heat balance between the thermal mass fluid element and a single solid element (Fig. 6):

$$
\dot{m} \cdot c_{p} \cdot\left(T_{\text {out }, f}-T_{\text {in }, f}\right)=A \cdot H T C \cdot\left(T_{w}-T_{\text {out }, f}\right)
$$

the outlet temperature of the fluid element results:

$$
T_{\text {out } f}=\frac{A \cdot H T C \cdot T_{w}+\dot{m} \cdot c_{p} \cdot T_{i n, f}}{A \cdot H T C+\dot{m} \cdot c_{p}}
$$

Imposing as $\mathrm{BC}$ a mass flow rate very small (not actually simulating an efflux but the very low thermal inertia) the outlet temperature of the fluid element will tend to the wall one: 


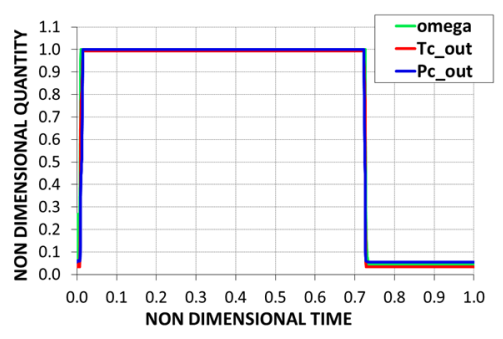

(a) Whole mission cycle.

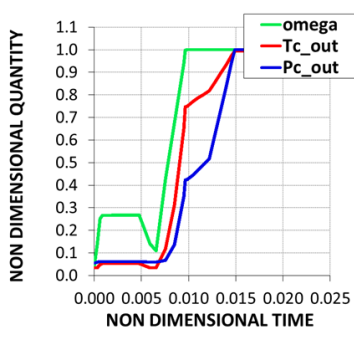

(b) Start-up phase.

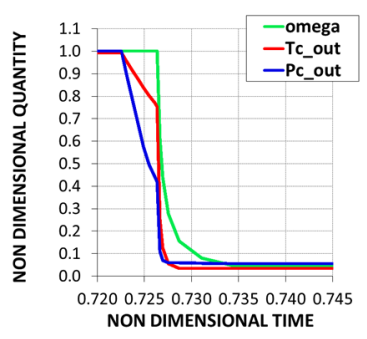

(c) Shutdown phase.

Fig. 7. Trends in time of rotational speed, temperature and pressure at the compressor exit.

$$
\lim _{\dot{m} \rightarrow 0} T_{\text {out }, f}=\lim _{\dot{m} \rightarrow 0} \frac{A \cdot H T C \cdot T_{w}+\dot{m} \cdot c_{p} \cdot T_{i n, f}}{A \cdot H T C+\dot{m} \cdot c_{p}}=T_{w}
$$

Therefore, with a very small mass flow rate, disregarding the value of the inlet temperature of the fluid element since mass flow contributions factors can be neglected, for $n$ solid surfaces involved, the fluid outlet temperature becomes an average of those of the solid walls:

$$
\lim _{\dot{m} \rightarrow 0} T_{\text {out }, f}=\frac{\sum_{i=1}^{n} A_{i} \cdot H T C_{i} \cdot T_{w, i}}{\sum_{i=1}^{n} A_{i} \cdot H T C_{i}}
$$

leading to a process of homogenization of the same wall temperatures through heat transfer.

Instead, in the case the thermal mass is subjected to a contribution of thermal power due for example to other SAS branches flowing inside the void region, the fluid element simulating the thermal mass is characterized by a mass flow rate equal to the sum of those of streams involved, and an additional heat load $\dot{Q}_{t m}$ is imposed in its inlet node, with a value corresponding to the sum of enthalpy contributions of the $n$ streams providing power:

$$
\dot{Q_{t m}}=\sum_{i=1}^{n} m_{i} \cdot c_{p} \cdot\left(T_{\text {out }, i}-T_{i n, t m}\right)
$$

where $m_{i}$ and $T_{\text {out }, i}$ are respectively the mass flow rate and the outlet temperature of the i-th stream flowing inside the cavity, and $T_{i n, t m}$ is the runtime solved inlet temperature of the fluid element associated to the thermal mass.

\section{Assessment of the proposed methodology}

The assessment of the thermo-mechanical setup of the procedure is carried out through its application to a real engine geometry under realistic conditions, comparing temperature results with those of a reference FEM code. The aim is to evaluate the transient thermal modelling capability of the procedure, testing the capacity of the solver to properly simulate the transient operation, according to the new features implemented in the CalculiX ${ }^{\circledR}$ code.

\subsection{Test case description}

The real engine model has been tested under real operation conditions. A representative cycle with base load conditions interposed between two fast ramps of acceleration and deceleration has been considered (by way of example, see time series reported in Fig. 7).

The solid axisymmetric model has been discretized into 126479 six nodes triangular elements with a total of 270186 nodes, and the setup followed what is stated in Section 3. For reasons of confidentiality, only a part of the whole engine simulated can be shown and discussed. Anyway, this portion provides a significative overview of the overall simulation since it is characterized by the presence of the main SAS devices (seals, preswirl components, 


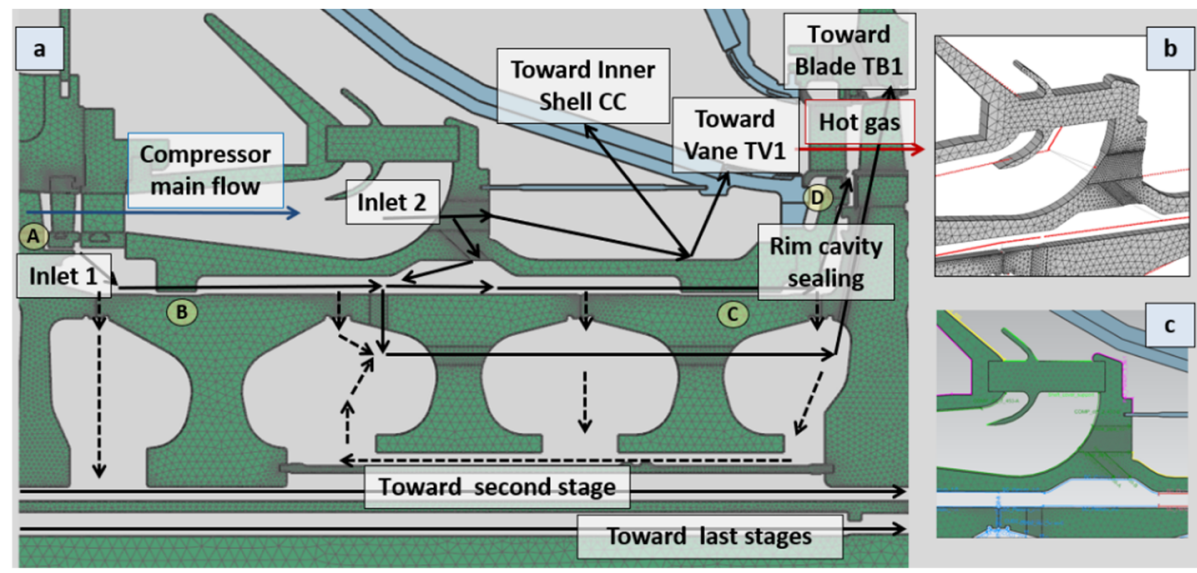

Fig. 8. Mesh and SAS main flows repartition; the four labyrinth seals (labelled A, B, C, D) of the model portion (a). Details of fluid mesh (b) and of BCs (c) complexity.

cavities, channels) and of a complete flow path of cooling/sealing air. In Fig. 8a, the main SAS branches along the portion are highlighted: from compressor seal regions, where the cooling flow is extracted for cooling of wheel spaces and for internal cooling of hot gas path components, the flow path extends along the shaft and stops at the first turbine stage.

In Fig. 8a main splits characterizing the main SAS flows of the SAS aerodynamic model are reported, but more complex distributions of flows are assigned in the thermal network (distinct from the aerodynamic one) used in the FEM model. In the thermal network, indeed, splits percentages of the main flows detected in the SAS aerodynamic solution are adopted in specific and more detailed thermal BCs (by way of example see details of Fig. 8b and 8c).

\subsection{Discussion of results}

The model has been run with the presented thermo-mechanical procedure based on CalculiX ${ }^{\circledR}$ and with a reference FEM solver. For reasons of brevity comparisons between the two solvers have been here reported in terms of temperature distributions in some relevant points of the solid domain, in order to establish an assessment of the consistency of the modelling adopted in the proposed procedure. Points have been chosen according to the proximity to zones of interest for the modelling. Reported temperatures are scaled with respect to a reference temperature $T_{\text {ref }}$ (approximately the maximum value detected at the compressor exit).

Fig. 9a and $9 \mathrm{~b}$ refer to points on blades, vanes and endwalls where BCs applied on them must be properly scaled according to requirements related to plane-axisymmetric interfaces. Fig. 9c and 9d show temperature trends of points positioned adjacent or overlooking a cavity enclosing a thermal mass. Fig. 9e and 9f refer to couples of nodes located on the opposite sides of the central labyrinth seals (B, C in Fig. 8a). Seals are important locations to monitor since their gap variability, redefining clearances due to the thermal expansion, can affect considerably the secondary air consumption and the possibility of detrimental interferences. Fig. $9 \mathrm{~g}$ and $9 \mathrm{~h}$ refer to nodes located in holes and channels. HP point is on the bores region collecting coolant towards the combustor and towards the first stage nozzles of the turbine. HSC point is on the preswirler region through which flow enters the plenum between the shaft and the shaft cover and joins the leakage flow from the last stage of the compressor (Inlet 1 Fig. 8a). Through the vertical and horizontal holes located in the $\mathrm{VH} 2$ point and $\mathrm{HH} 2$ point regions, respectively, air goes toward the cooling system of the first stage blades. These points are located on non-axisymmetric features, in static and rotating reference frames, modelled using an equivalent thickness of plane elements and proper scaling of quantities. 


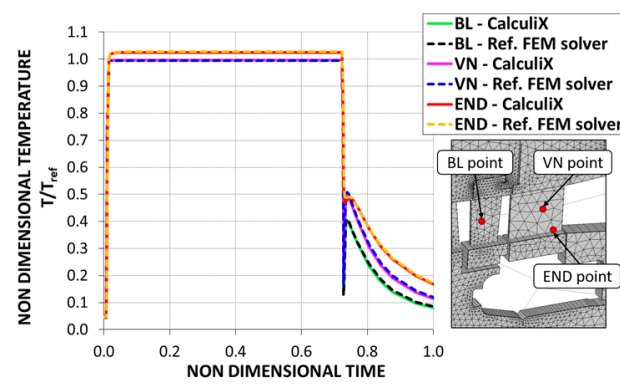

(a) Points located on blades and vanes.

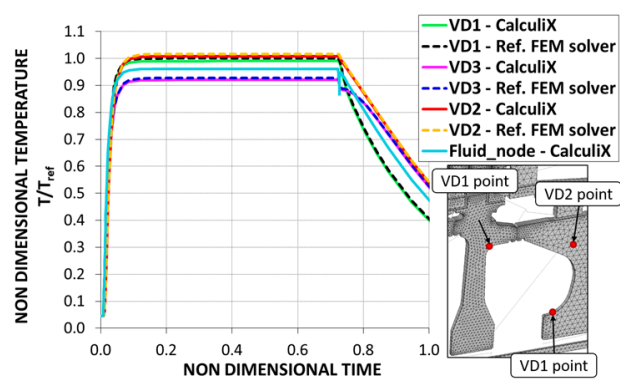

(c) Points adjacent to cavities.

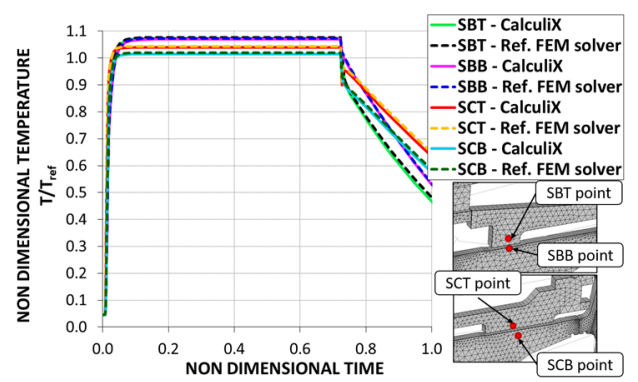

(e) Points on opposite sides of labyrinth seals.

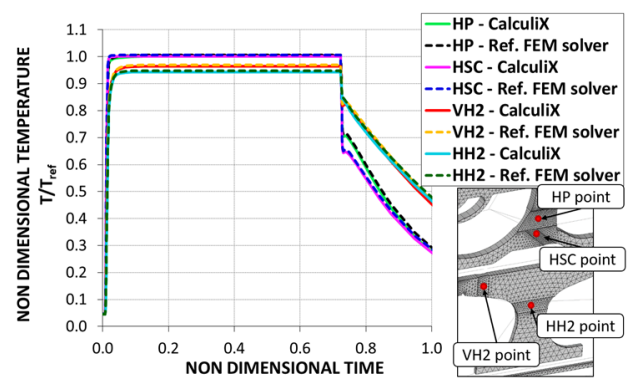

(g) Points inside holes regions.

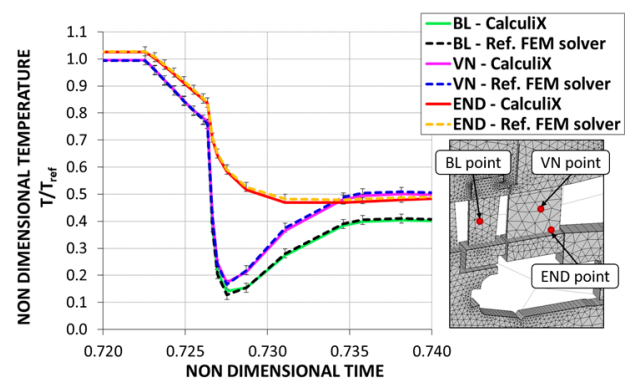

(b) Shutdown details of blades and vanes points.

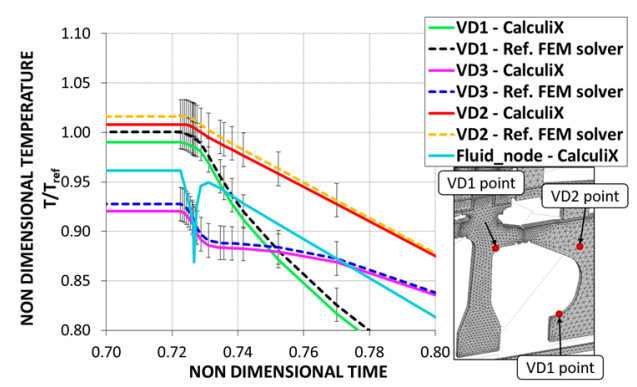

(d) Shutdown details of cavity points.

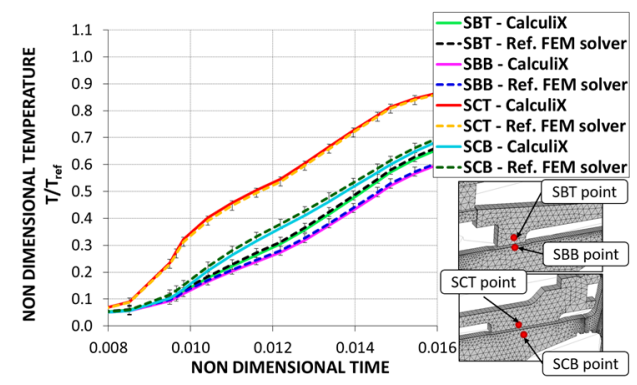

(f) Sturt-up details of points on seals.

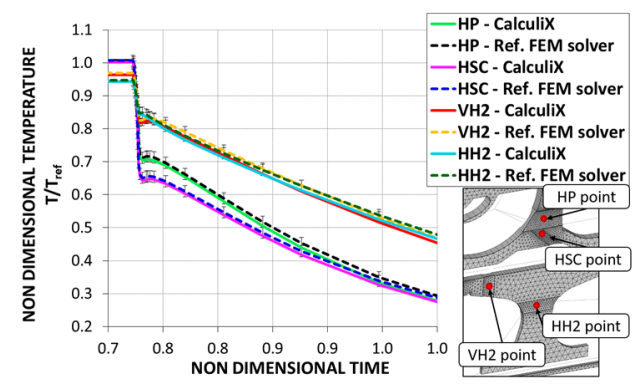

(h) Shutdown details of points on holes.

Fig. 9. Non dimensional temperature trends versus non dimensional time.

According to results of Fig. 9 the procedure demonstrates to be able to follow the transient operation, catching a coherent behaviour of the thermal model according to the engine cycle and the physic of the problem. As temperature trends suggest, due to the imposed mission cycle, there is a sudden rise in temperature in the start-up phase followed by baseload conditions, concluding then with a shutdown phase characterized by a quick temperature 
reduction during the firing cut off. During this phase mass flow entering is very small and a slight increase in the fluid temperature is generally observed due to the heat received from the metal, after the engine is turned off. The effect is visible on the pick of Fig. 9d on the fluid node simulating the thermal mass associated to the cavity at stake. Similarly, while flows are quite exhausted the predominant phenomenon on the solid domain is the conduction of the heat accumulated in the rotor till that moment, so the effect of the rotor thermal inertia affects components like blades which are involved in a temporary increase as it can be observed in Fig. 9a and 9b. After that phase, a gradual decrease in temperatures is observed. Points located in more internal zones of the rotor (Fig. 9c, 9e, 9g) where the thermal inertia is higher, are subjected to smoother variations.

Results summarized in Fig. 9 report an overall good agreement between the predictions of the proposed methodology and the reference FEM solver, showing differences not exceeding $7.5^{\circ} \mathrm{C}$ (value used for all error bars displayed). Observed differences even in the case of the highest deviations (e.g. Fig. 9f and 9h), show in any case a concordance of trends, and the detected shifting is probably due to a not fully equivalent level of discretization in time of the two solvers, i.e. in the way they adjust the time step runtime, as preliminary investigations (not subject of this work) suggest. The significant agreement between the predictions of the two solvers ensures the coherence of the procedure with the phenomena involved and attests the consistency of the CalculiX ${ }^{\circledR}$ based thermo-mechanical procedure.

\section{Conclusions}

In this work a transient thermo-mechanical methodology for gas turbine WEM applications and its assessment have been presented. The procedure is based on a FEM-fluid network coupling approach through a customized version of the open source code Calculi ${ }^{\circledR}$.

A 2D axisymmetric model of the whole cross section of the engine is coupled to a thermal fluid network. Mass flow rates and pressures obtained from a prior SAS aerodynamic solution are applied in the thermal network available in the FEM model, providing an intrinsic coupling of the fluid-solid interactions in the thermo-mechanical solution, with the evaluation of the fluid-metal temperatures and displacements by means of a single model. Using a prior aerodynamic solution separated from the thermo-mechanical analysis allows to obtain a modular structure of the thermo-mechanical tool and to maintain two different levels of discretization of the fluid network mesh, satisfying different requirements of coarseness of the fluid mesh in the two different analyses.

The modularity and the open source peculiarities of CalculiX ${ }^{\circledR}$ free the user to insert desired customizations and to adapt the internal solver according to the requirements of specific and more complex procedures in which the thermo-mechanical model can be included, making the tool very versatile both in design and analysis phase. To this end, an overview of its application in a coupled WEM aero-thermo-mechanical methodology developed by the authors has also been recalled in this work.

With the aim to propose a comprehensive description of the modelling features for the engine transient thermo-mechanical characterization in WEM applications, the main assumptions, models and calculation setup have been discussed.

Then the proposed procedure has been tested on a real whole engine geometry under a transient operating cycle. Results have been compared with those of a reference FEM solver, showing a good agreement and attesting the solver capabilities and the coherence of the modelling features introduced.

The authors would like to acknowledge Dr. G. Dhondt from MTU Aero Engines Munich for his precious advices and to thank Mr. Carlos Simon-Delgado and Mr. Luca Bordo from Ansaldo Energia for their help in carrying out this project. 


\section{References}

[1] V. Ganine, N. Hills, M. Miller, C. Barnes, S. Curzons, L. Turner, P. Smout, Proceedings of ASME Turbo Expo 2015: Turbine Technical Conference and Exposition, 5C, GT2015-43406 (2015)

[2] A. Peschiulli, D. Coutandin, M. D. Cioppo, M. Damasio, Proceedings of ASME Turbo Expo 2009: Power for Land, Sea, and Air, 3, GT2009-59875 (2009).

[3] G. Tondello, W. Boruszewski, F. Mengele, M. Assato, S. Shimizu, S.Ziegler, Proceedings of the ASME Turbo Expo 2012: Turbine Technical Conference and Exposition, 4, GT2012-69924 (2012)

[4] V. V. Reddy, K. Selvam, R. De Prosperis, Proceedings of ASME Turbo Expo 2016: Power for Land, Sea, and Air, 5C, GT2016-56601 (2016)

[5] Y. Muller, Proceedings of ASME Turbo Expo 2008: Power for Land, Sea, and Air, 4, GT2008-50078 (2008)

[6] Y. Muller, Proceedings of ASME Turbo Expo 2009: Power for Land, Sea, and Air, 3, GT2009-59104 (2009)

[7] S. Giuntini, A. Andreini, B. Facchini, M. Mantero, M. Pirotta, S. Olmes, T. Zierer, Proceedings of the ASME Turbo Expo 2017: Turbomachinery Technical Conference and Exposition, 5B, GT2017-64512 (2017)

[8] S. Giuntini, A. Andreini, G. Cappuccini, B. Facchini, Energy Procedia, 126, 746753 (2017)

[9] S. Giuntini, A. Andreini, B. Facchini, Proceedings of the ASME Turbo Expo 2019: Turbomachinery Technical Conference and Exposition, 5A, GT2019-91278 (2019)

[10] V. Ganine, U. Javiya, N. Hills, J. Chew, Proceedings of the ASME Turbo Expo 2012: Turbine Technical Conference and Exposition, 4, GT2012-68989 (2012)

[11] G. Dhondt, CalculiX CrunchiX USER'S MANUAL version 2.11, http://www.calculix.de/ (2011)

[12] G. Dhondt, The Finite Element Method for Three-dimensional Thermomechanical Applications, (John Wiley \& Sons, 2004) 